\title{
Heavy Quarkonium Decays on and off the Lattice
}

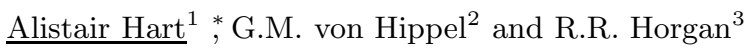 \\ 1-University of Edinburgh - School of Physics \\ King's Buildings, Edinburgh EH9 3JZ - United Kingdom \\ 2-University of Regina - Department of Physics \\ Regina, SK, S4S 0A2 - Canada \\ 3-University of Cambridge - DAMTP \\ CMS, Cambridge CB3 0WA - United Kingdom
}

I review recent progress in understanding radiative transitions in heavy quarkonium, both on and off the lattice, and discuss our recent leptonic width matching calculation.

\section{Introduction}

In my conference talk (available at [1]), I reviewed papers of Dudek, Edwards and Richards $[2,3]$, of Lansberg and Pham [4], of Gao, Zhang and Chao [5] and of Oliveira and Coimbra [6]. As these are discussed elsewhere in these proceedings, I here concentrate on our calculation of the leptonic widths of heavy quarkonia [7].

Leptonic widths of heavy quarkonia such as the $\Upsilon$ or the $J / \psi$ are an important test of electroweak Standard Model in the heavy quark sector: heavy particles should be sensitive to possible new physics at or above the electroweak scale. Leptonic decays have experimentally clean signatures. Moreover, ratios of leptonic widths can be measured to good accuracy both experimentally and on the lattice, providing a high precision test of lattice techniques.

Here we address how to improve the precision of current lattice predictions [8] to match that of experimental results [9]:

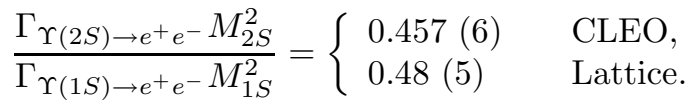

\section{Matching S-wave decays between NRQCD and QCD}

The leptonic width of a heavy quarkonium $\bar{Q} Q$ state of mass $M_{\bar{Q} Q}$ is given by

$$
\Gamma_{\bar{Q} Q \rightarrow l^{+} l^{-}}=\frac{8 \pi}{3 M_{\bar{Q} Q}}\left|\left\langle 0\left|\mathbf{J}^{Q C D}\right| \bar{Q} Q\right\rangle\right|^{2} e_{Q}^{2} \alpha_{e m}^{2}
$$

with nonperturbative QCD contributions coming from the matrix element $\left\langle 0\left|\mathbf{J}^{Q C D}\right| \bar{Q} Q\right\rangle$. Unfortunately, it is not possible to simulate heavy $b$ quarks directly on a lattice (with spacing typically $a \simeq 0.1 \mathrm{fm}$ ) due to their short Compton wavelengths. We must use an effective theory, such as NRQCD, and calculate the desired QCD matrix element from a set of NRQCD matrix elements which can be measured on the lattice:

$$
\left\langle 0\left|\mathbf{J}^{Q C D}\right| \bar{Q} Q\right\rangle=\sum_{i} a_{i}\left\langle 0\left|\mathbf{J}_{i}^{N R Q C D}\right| \bar{Q} Q\right\rangle
$$

\footnotetext{
${ }^{*}$ U.K. Royal Society University Research Fellow
} 
In this paper we determine the matching coefficients $a_{i}$ for S-wave decays, with NRQCD currents $\mathbf{J}_{i}^{N R Q C D}=\boldsymbol{\sigma}\left(\frac{\Delta^{2}}{M^{2}}\right)^{i}$ (which vary as $v^{2 i}$ with the heavy quark velocity at tree level).

Discretisation removes high momentum modes, so it is reasonable to expect that we can compute the matching coefficients perturbatively, by expanding both the coefficients and the matrix elements and matching order by order in $\alpha_{s}$ :

$$
a_{i}=\sum_{n} \alpha_{s}^{n} a_{i}^{(n)} \quad\langle 0|\mathbf{J}| \bar{Q} Q\rangle=\sum_{n} \alpha_{s}^{n}\langle 0|\mathbf{J}| \bar{Q} Q\rangle^{(n)}
$$

We calculate $a_{0,1,2}^{(0)}$ and $a_{0,1}^{(1)}$, giving an accuracy of $\mathcal{O}\left(\alpha_{s}, \alpha_{s} v^{2}, v^{4}\right)$. In the $\Upsilon$ system, $v^{2} \sim$ $\alpha_{s} \sim 10 \%$ suggesting that to achieve $\sim 1 \%$ accuracy, we would need to go to $\mathcal{O}\left(\alpha_{s}^{2}, \alpha_{s} v^{2}, v^{4}\right)$, hence requiring two-loop $a_{0}^{(2)}$. In matrix element ratios, however, we need only $b_{1,2}=a_{1,2} / a_{0}$ and such terms cancel. Our calculation thus give $\sim 1 \%$ accuracy on the ratio.

We work in the Breit frame, where the decaying meson is stationary and the quark has momentum $p^{\mu}=(i E, 0,0, M v)$, use $v$ as the non-relativistic expansion parameter (exact at the order to which we are working) and treat the quarks as being on-shell (which can also be shown to be justified). Our gauge and fermion actions are chosen to be the same as are used in current lattice simulations. The improved NRQCD action is

$$
\mathcal{S}_{\mathrm{NRQCD}}=\sum_{x, t} \psi^{\dagger} \psi-\psi^{\dagger}\left(1-\frac{a \delta H}{2}\right)\left(1-\frac{a H_{0}}{2 n}\right)^{n} U_{4}^{\dagger}\left(1-\frac{a H_{0}}{2 n}\right)^{n}\left(1-\frac{a \delta H}{2}\right) \psi .
$$

where $n$ is a stability parameter for the euclidean-space Schrödinger equation, which must satisfy $n \geq 3 /(M a)$ for numerical stability. For the gauge fields, we use a Symanzik improved action with tadpole improved links.

The Feynman rules for such actions are extremely complicated, with 8000 terms in the $Q \bar{Q} g$ vertex and 70000 for the $\mathcal{O}(a) Q \bar{Q} g g$. For this reason, we have developed HiPPy, a flexible, automated tool for generating Feynman rules from lattice actions [10]. It incorporates automatic differentiation techniques [11] to calculate the derivatives of the complicated Feynman diagrams. Freely available, HiPPy has also been used in a number of recent calculations $[12,13,14]$.

\subsection{Matching at tree level}

At tree-level, the relevant matrix elements are given by

$$
\begin{aligned}
\left\langle 0\left|\mathbf{J}^{Q C D}\right| \bar{Q} Q\right\rangle^{(0)} & =\bar{v}(-\mathbf{p}) \boldsymbol{\gamma} u(\mathbf{p})=\chi^{\dagger} \boldsymbol{\sigma}\left(\frac{2}{3}+\frac{M}{3 E}\right) \psi \\
\left\langle 0\left|\mathbf{J}_{i}^{N R Q C D}\right| \bar{Q} Q\right\rangle^{(0)} & =g_{i}(v) \chi^{\dagger} \boldsymbol{\sigma} \psi
\end{aligned}
$$

where

$$
\begin{aligned}
& g_{0}(v)=1, \quad g_{1}(v)=-\frac{4}{(M a)^{2}} \sin ^{2}\left(\frac{a M v}{2}\right)=v^{2}+\mathcal{O}\left(v^{4}\right) \\
& g_{2}(v)=\frac{4}{(M a)^{2}}\left[4 \sin ^{2}\left(\frac{a M v}{2}\right)-\sin ^{2}(a M v)\right]=v^{4}+\mathcal{O}\left(v^{6}\right)
\end{aligned}
$$



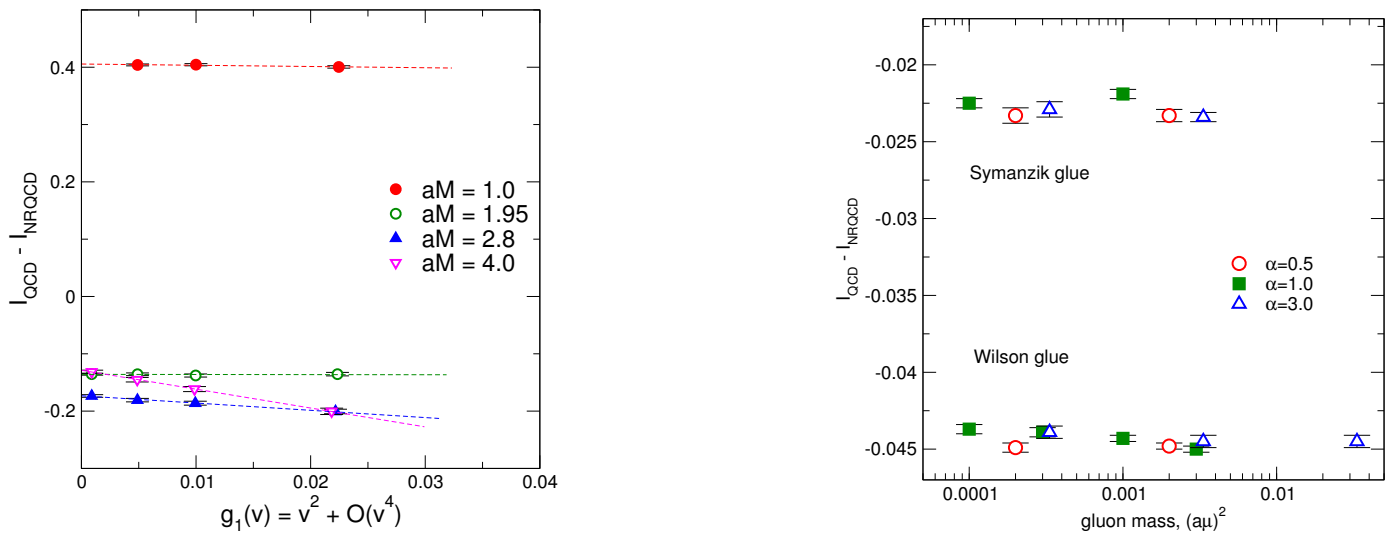

Figure 1: Left: numerical results with fits for $\left.a_{0,1}^{(1)}\right)$; right: results in different gauges vs. the infrared gluon mass, showing gauge and gluon mass independence.

Expanding these matrix elements in powers of $v^{2}$, we determine $a_{i}^{(0)}$ to match:

$$
a_{0}^{(0)}=1, \quad a_{1}^{(0)}=\frac{1}{6}, \quad a_{2}^{(0)}=\frac{1}{8}-\frac{(a M)^{2}}{72} .
$$

\subsection{Matching to one-loop order}

Expanding the matching condition to first order in $\alpha_{s}$ gives

$$
\sum_{i} \underbrace{a_{i}^{(1)}}_{\text {wanted }} \overbrace{\left\langle 0\left|\mathbf{J}_{i}^{N R Q C D}\right| \bar{Q} Q\right\rangle^{(0)}}^{\text {known functions of } v}=\overbrace{\left\langle 0\left|\mathbf{J}^{Q C D}\right| \bar{Q} Q\right\rangle^{(1)}}^{I_{Q C D}}-\overbrace{\sum_{i} a_{i}^{(0)}\left\langle 0\left|\mathbf{J}_{i}^{N R Q C D}\right| \bar{Q} Q\right\rangle^{(1)}}^{I_{N R Q C D}}
$$

Both the QCD and the NRQCD matrix elements on the right-hand side contain odd powers of $v$ coming from the Coulomb-exchange singularity; however, only even powers of $v$ are available for matching on the left-hand side, so the odd powers must cancel exactly.

In fact, the odd powers of $v$ are a purely infrared phenomenon, and are known exactly:

$$
I_{o d d}=\frac{h(v)}{12 v}=-\Im\left\{\frac{4}{3} \int \frac{d^{4} k}{(2 \pi)^{4}} \frac{h(v)}{\left(\boldsymbol{k}^{2}+\mu^{2}\right)\left(i k_{0}-\frac{\boldsymbol{k}^{2}+2 \boldsymbol{k} \cdot \boldsymbol{p}}{2 M}\right)\left(i k_{0}+\frac{\boldsymbol{k}^{2}+2 \boldsymbol{k} \cdot \boldsymbol{p}}{2 M}\right)}\right\}
$$

where $h(v)$ is a known even function of $v$. We can hence analytically subtract the odd powers from both QCD and NRQCD by rearranging the right-hand side as

$$
I_{Q C D}-I_{N R Q C D}=\left(I_{Q C D}-I_{o d d}\right)-\left(I_{N R Q C D}-I_{\text {in }}\right)+I_{\text {out }}
$$

where we have split $I_{\text {odd }}=I_{\text {in }}+I_{\text {out }}$, inside and outside the Brillouin zone. The term $\left(I_{Q C D}-I_{o d d}\right)$ is known analytically, while the other terms are calculated numerically using farmed VEGAS on the CCHPCF SunFire Galaxy class computer. We find the matching coefficients by fitting results for various $v$ with $\left(I_{Q C D}-I_{N R Q C D}\right)(v)=a_{0}^{(1)}-a_{1}^{(1)} g_{1}(v)$. 


\begin{tabular}{|llllll|}
\hline$M_{0} a$ & $n$ & $a_{0}^{1}$ & $a_{1}^{1}$ & $b_{1}^{1}$ & $b_{2}^{0}$ \\
\hline 4.0 & 2 & $-0.1288(27)$ & $-3.32(29)$ & $-3.30(30)$ & -0.0972 \\
2.8 & 2 & $-0.1732(21)$ & $-1.35(22)$ & $-1.32(22)$ & 0.0161 \\
1.95 & 2 & $-0.1358(16)$ & $0.26(17)$ & $0.14(17)$ & 0.0722 \\
1.0 & 4 & $0.4056(20)$ & $-0.50(17)$ & $-0.56(17)$ & 0.1111 \\
\hline
\end{tabular}

Table 1: The matching coefficients, as a function of the bare heavy quark mass. Note that $a_{0}^{(0)}=1, a_{1}^{(0)}=b_{1}^{(0)}=\frac{1}{6}$, and that there is no subtraction to prevent mixing down.

\section{Results and conclusions}

We have calculated matching coefficients at a number of quark masses corresponding to the bottom and charm quarks on the MILC improved staggered ensembles. We have performed extensive tests of gauge invariance, infrared regulator independence, and agreement with known results for $a_{0}^{(1)}$ at $v=0$ for simpler NRQCD actions. Our results are shown in Fig. 1, as well as a plot showing the gauge and regulator independence of our results. Our final results for the matching coefficients are given in Table 1 , and are currently being combined with lattice NRQCD matrix elements to predict the leptonic widths.

\section{Acknowledgments}

We thank G.P. Lepage and C.T.H. Davies for useful discussions and the Cambridge-Cranfield High Performance Computing Facility. G.M.v.H. thanks the Canadian Natural Sciences and Engineering Research Council and the Government of Saskatchewan for financial support.

\section{References}

[1] Slides: http://indico. cern. ch/contributionDisplay . py? contribId=209\&sessionId=5\&conf Id=9499

[2] J. J. Dudek and R. G. Edwards, Phys. Rev. Lett. 97, 172001 (2006), [hep-ph/0607140].

[3] J. J. Dudek, R. G. Edwards and D. G. Richards, Phys. Rev. D73, 074507 (2006), [hep-ph/0601137].

[4] J. P. Lansberg and T. N. Pham, Phys. Rev. D74, 034001 (2006), [hep-ph/0603113]; Phys. Rev. D75, 017501 (2007), [hep-ph/0609268].

[5] Y.-J. Gao, Y.-J. Zhang and K.-T. Chao, Commun. Theor. Phys. 46, 1017 (2006), [hep-ph/0606170]; Chin. Phys. Lett. 23, 2376 (2006), [hep-ph/0607278]; hep-ph/0701009.

[6] O. Oliveira and R. A. Coimbra, hep-ph/0603046.

[7] A. Hart, G. M. von Hippel and R. R. Horgan, Phys. Rev. D75, 014008 (2007), [hep-lat/0605007]; PoS LAT2006, 098 (2006), [hep-lat/0609002].

[8] A. Gray et al., Phys. Rev. D72, 094507 (2005), [hep-lat/0507013].

[9] CLEO, J. L. Rosner et al., Phys. Rev. Lett. 96, 092003 (2006), [hep-ex/0512056].

[10] A. Hart, G. M. von Hippel, R. R. Horgan and L. C. Storoni, J. Comput. Phys. 209, 340 (2005), [hep-lat/0411026].

[11] G. M. von Hippel, Comput. Phys. Commun. 174, 569 (2006), [physics/0506222]; arXiv:0704.0274.

[12] I. T. Drummond, A. Hart, R. R. Horgan and L. C. Storoni, Phys. Rev. D66, 094509 (2002), [heplat/0208010]; Nucl. Phys. Proc. Suppl. 119, 470 (2003), [hep-lat/0209130]; Phys. Rev. D68, 057501 (2003), [hep-lat/0307010].

[13] A. Hart, R. R. Horgan and L. C. Storoni, Phys. Rev. D70, 034501 (2004), [hep-lat/0402033].

[14] Z. Hao, G. M. von Hippel, R. R. Horgan, Q. J. Mason and H. D. Trottier, arXiv:0705.4660. 